\title{
Special Section:
}

\section{Understanding analytical systems technology for selective analysis}

\section{Introduction}

\author{
T. D. Geary \\ Institute of Medical and Veterinary Science, Adelaide, South Australia, Australia
}

The members of the International Federation of Clinical Chemistry Expert Panel on Instrumentation made a decision to present a series of symposia to discuss and allay fears caused by the advances in technology which have led to the development of the 'black box'.

The Organizing Committee of the International Congress of Clinical Chemistry (The Hague, 1987) was approached and time was allocated for the first of these symposia. The symposium was structured to explain in detail the functions of the 'black box', as well as its components, and had the appropriate title Technology for Selective Analysis. Selective analysers embody many of the components which contribute to the feeling of uncertainty, referred to as the 'black box syndrome'.

The 'black box' can be divided into its individual components as indicated for an analytical instrument in figure 1. It is important to be conversant with the operation of the individual parts in order to understand the functioning of the total.

\begin{tabular}{l|l|l} 
& SAMPLING \\
& MIXING \\
& INCUBATING \\
& MEASURING \\
Figure 1. & CALGULATING \\
DISPLAYING & $\rightarrow$ ANALYTICAL
\end{tabular}

Before this task can be tackled it is necessary to provide some relevant definitions. The first of these being the definition of an analytical instrument, which is a device or a combination of devices used to carry out an analytical process. This is illustrated in figure 1. The second is an analytical system which is a system of devices with process control. Reagents are considered to be an integral part of an analytical system (figure 2):

ANALYTICAL INSTRUMENT

Figure 2.

REAGENTS
ANALYTICAL
SYSTEM

The development of the Skeggs analytical instrument was a significant advance in clinical laboratory medicine. It brought together mixing, sampling, separating, measuring and displaying devices in a sequence which mimicked the manual operation in a manner which provided increased sampling rate and led to decrease in the imprecision of the analytical methods in use. The instrument relied upon continuous flow air segmentation to transport the reaction mixture. Since that time, there have been other transport systems developed, which have provided opportunities in other areas of the clinical laboratory. These innovations have led to the systems being more operator independent - but with an increase in complexity. This increase in complexity and lack of involvement in the assay contributes to the worries associated with the 'black box'.

The symposium considered the following topics:

(1) General principles for the classification of analysers.

(2) Selectivity and random access in automatic analysers.

(3) System control: sampling, diluting, dispensing, and temperature control.

(4) System control: optics and electronic system control.

(5) Transport and carry-over.

(6) Organizational aspects of selective analysers; choosing an instrument appropriate to the laboratory's requirements. 


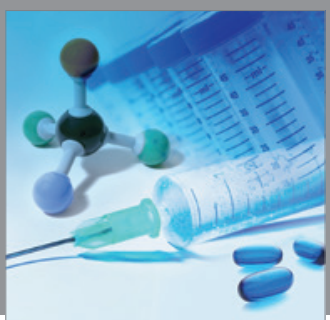

International Journal of

Medicinal Chemistry

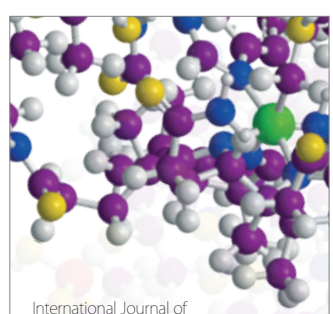

Carbohydrate Chemistry

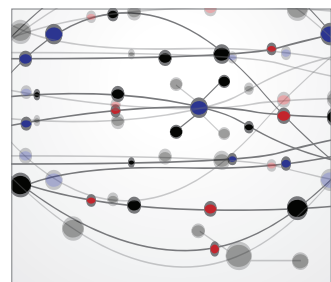

The Scientific World Journal
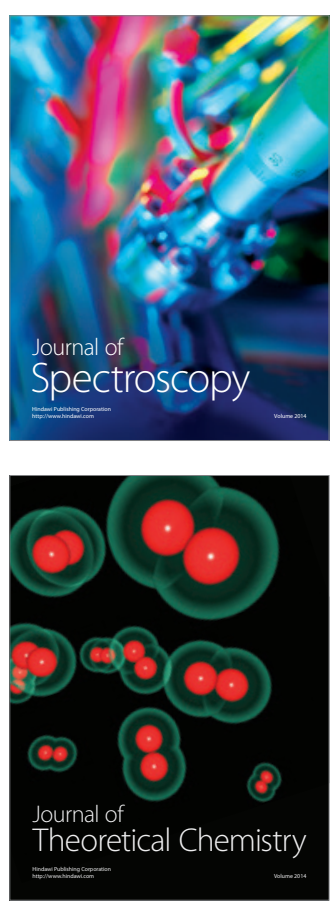
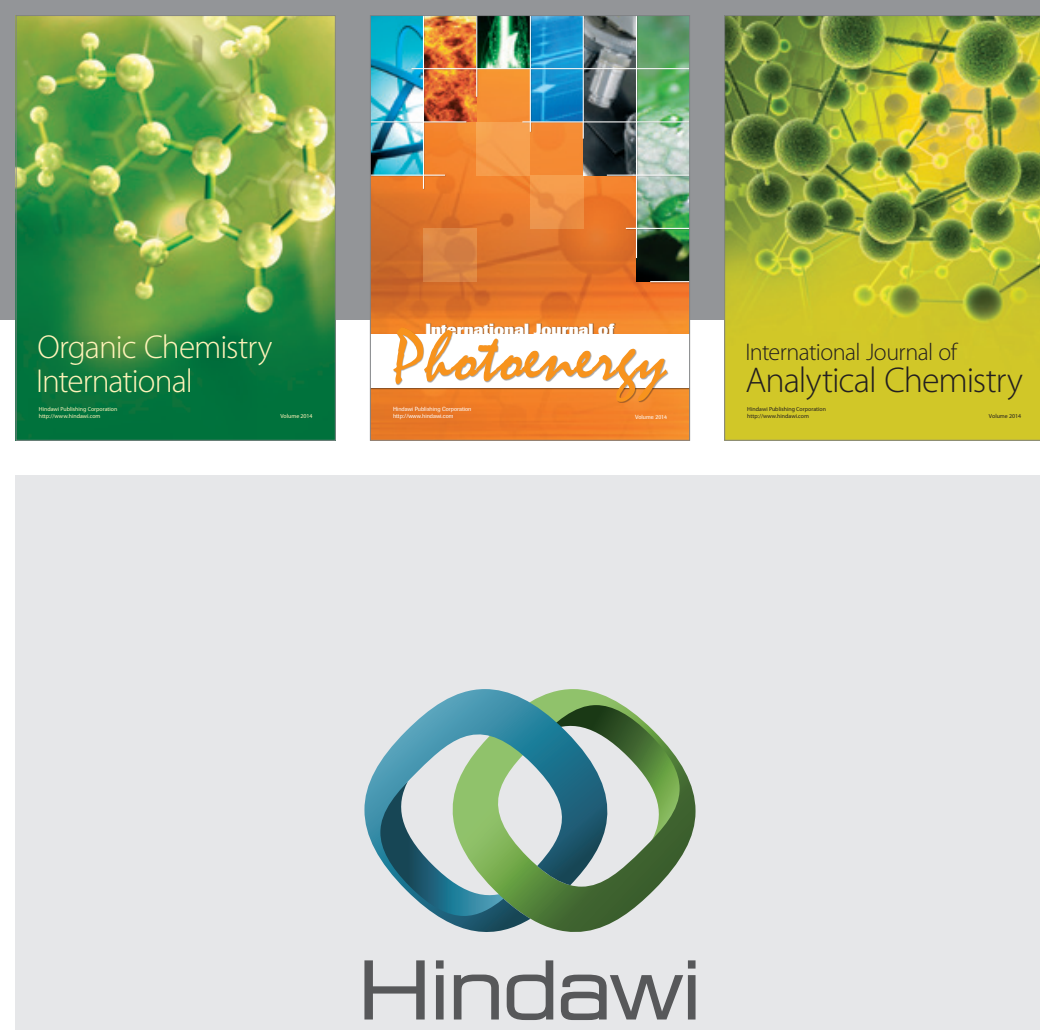

Submit your manuscripts at

http://www.hindawi.com
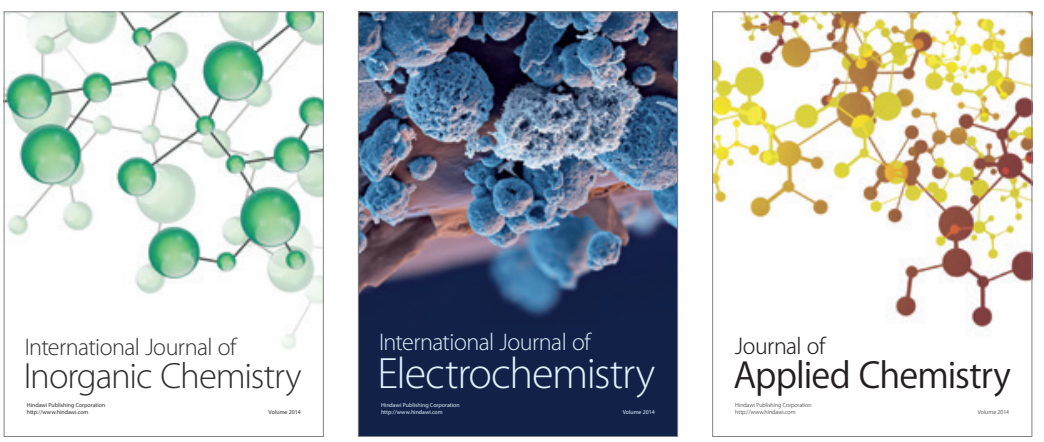

Journal of

Applied Chemistry
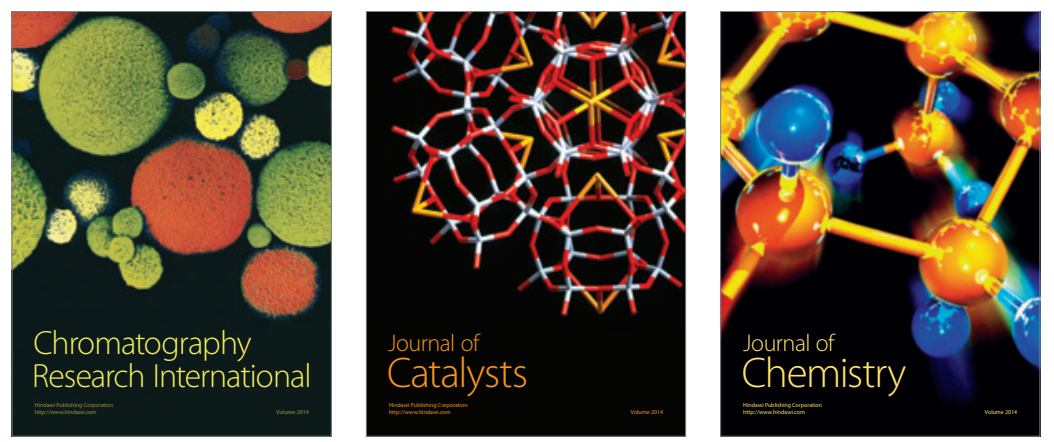
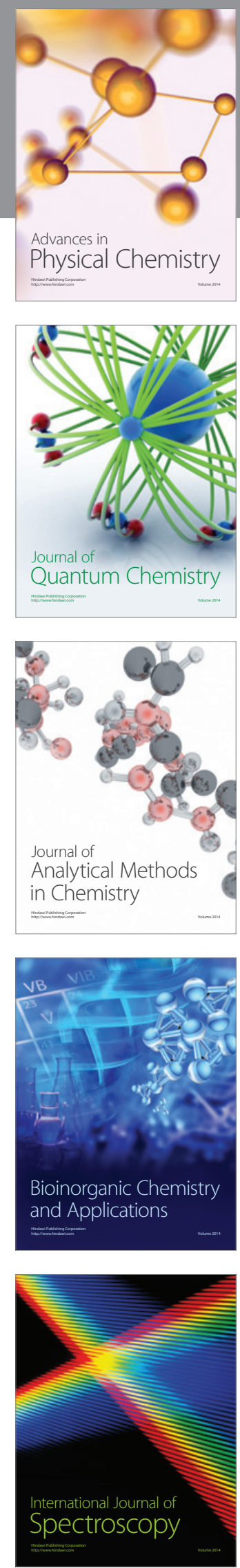